Caballero-García, P., Jiménez-Martínez, M.P. \& Guillén-Tortajada, E. (2019). Aprender a emprender bajo el binomio familia-escuela. Revista Electrónica Interuniversitaria de Formación del Profesorado, 22(3), 139-154.

DOI: http://dx.doi.org/10.6018/reifop.22.3.389611

\title{
Aprender a emprender bajo el binomio familia-escuela*
}

Presentación Caballero-García, María Pilar Jiménez Martínez, Esmeralda Guillén Tortajada.

Facultad de Educación y Salud. Universidad Camilo José Cela. Madrid

\section{Resumen}

La discusión sobre si escuela y familia influyen en la formación continua del discente para el desarrollo laboral, ha despertado gran interés social. La competencia emprendedora no se trabaja suficientemente desde la escuela y, sin embargo, facilita la inserción laboral futura. Defendemos su impulso conjunto desde el núcleo familiar y la escuela, involucrando a la comunidad. La investigación demuestra que estos programas educativos integrados estimulan el éxito académico, la inclusión social y la inserción laboral. El artículo revisa la literatura sobre el tema, ofreciendo una propuesta de práctica exitosa de desarrollo de la competencia emprendedora, basada en la sinergia familia-escuela.

\section{Palabras clave}

Competencia emprendedora; participación familiar; participación comunitaria; colaboración familia-escuela.

\section{Learning to undertake under the family-school binomial}

\section{Abstract}

The discussion about how education and family influence the continuous training of the student for the development of the labour market, has aroused great social interest. The entrepreneurial competence is not worked enough from the school environment and, however, facilitates the future employability. We advocate for the joint impulse of the family and the school nucleus, involving the community. Researches show that these

\section{Contacto:}

Presentación A. Caballero García. Facultad de Educación. Universidad Camilo José Cela. C/ Castillo de Alarcón, 49 • Urb. Villafranca del Castillo · 28692 Madrid. Email: pcaballero@ucjc.edu

* Trabajo desarrollado por el Gdl ASE, dentro del Proyecto I+D titulado HACKER \& HAPPY. Originales, Audaces e inteligentes, № expediente: 2015-22, financiado por la Universidad Camilo José Cela, en su IV Convocatoria de Ayudas a la Investigación 
integrated educational programs stimulate academic success, social inclusion and employment insertion. The article reviews the literature about the subject, offering a successful practice proposal of developing entrepreneurial competence, based on familyschool synergy.

\section{Key words}

Entrepreneurial competence; family participation; community participation; family-school collaboration.

\section{Introducción}

La iniciativa emprendedora es uno de los principales impulsores de la innovación, la competitividad y el desarrollo económico y social de un país (De la Torre et al., 2015). Estudios relativamente recientes (Martínez-Méndez, 2007; Uribe, Valenciano y Bonilla, 2013, entre otros) defienden que el espíritu emprendedor es enseñable y señalan la importancia de incluirlo en las aulas. Queda en manos de los docentes el desarrollo de estas destrezas (Caballero-García, Jiménez y Guillén, 2017a).

La educación puede contribuir enormemente a la creación de una cultura emprendedora (Arnaiz, 2015), empezando desde la escuela y llegando hasta la Universidad, a través de un marco integrado y coherente (Navarro y Torregrosa, 2012; Sobrado y Fernández, 2010). Y puede hacerlo fomentando una actitud favorable hacia ella, aumentando la sensibilización hacia salidas profesionales y proporcionando competencias empresariales para este fin (Martínez Méndez, 2007). "Las cualidades personales pertinentes para el espíritu empresarial, como la creatividad, la capacidad de iniciativa y el sentido común, pueden ser útiles para todos, tanto en la actividad laboral como en la vida cotidiana" (Uribe et al., 2013, p. 653). Merece la pena, por tanto, prestarle atención educativa, y no solo puntual, sino adecuadamente planificada y sistematizada.

\footnotetext{
Las personas que mayor impacto social provocarán en este siglo XXI están hoy en proceso de formación. En la universidad, en colegios e institutos, ahí encontramos a los hombres y mujeres que moverán el mundo. Por este motivo es clave que las instituciones educativas hagan eco de esta demanda social y fomenten al menos el desarrollo de unas claves mínimas para la generación de la cultura del emprendimiento (Béjar, 2017, p. 65).
}

Para trabajar el espíritu y la cultura del emprendimiento en la escuela, base fundamental y complementaria del desarrollo de la capacidad emprendedora, es necesario conocer la cultura escolar, pues permite identificar las fortalezas y debilidades propias, ayuda a distanciarse de las rutinas y creencias personales, y promueve formas creativas y alternativas de percibir y proponer innovaciones (García-Zárate, 2013). La educación para el emprendimiento tiene en cuenta en qué medida son incorporados a los sistemas educacionales y de capacitación, el conocimiento, las destrezas y habilidades emprendedoras (León y Barrera, 2017). Por eso es importante un movimiento investigador que genere y promueva iniciativas para el emprendimiento en nuestras aulas, comprometido con la profesionalización de la formación que se imparte, alineado con el 
tejido productivo y las demandas sociales presentes y futuras (Caballero-García et al., 2017a, 2017b), que mida resultados y propicie procesos de mejora constantes.

En este artículo, respaldamos la importancia de acompañar al estudiante en la adquisición de la competencia emprendedora en la escuela y desde etapas infantiles, e invitamos a hacerlo en colaboración con la familia. "Es necesario puntualizar el papel trascendental que juegan la familia, la escuela y las instituciones y la incidencia de estas estructuras en la construcción del sujeto" (Orrego, 2009, pp. 245-246). Debemos remontarnos a un proceso que debe desarrollarse a lo largo de la vida, desde los primeros años. Es aquí donde entra a formar parte de manera significativa la familia, que posteriormente deberá coordinarse con el contexto escolar (Santamaría, Moreno, Torres y Cadrazco, 2013) y la sociedad para este fin.

La formación del discente en la escuela debe ir ligada a un mayor acceso a contextos reales, como la familia, la comunidad, el trabajo o la propia empresa, en los que los niños y jóvenes puedan poner en práctica sus competencias (Jaramillo, 2008). Estudios recientes destacan la estrecha conexión entre la intención de emprender y los entornos cercanos al emprendedor, como son la familia y la escuela (Jaén, Moriano, y Liñán, 2013). La unión núcleo familiar-escuela influye en la formación continua del discente y su desarrollo como emprendedor en el mundo laboral.

García-Sanz, Hernández-Prados, Parra-Martínez y Gomariz-Vicente (2016) señalan que la implicación de los padres en la educación de los hijos es un aspecto esencial. Las familias son un espacio privilegiado para la educación de las nuevas generaciones. Serán, por tanto, un aliado fundamental en el desarrollo de la cultura y la competencia emprendedora. El alumno adquiere de la familia, la escuela y del entorno cercano, sus primeras experiencias sobre lo que significa ser emprendedor (Romero y Espasandín, 2016).

Proyectos como el "Harvard Family Research" aportan resultados de participación de los padres en la escuela, desde la etapa infantil hasta la universitaria, y todos ellos apuntan al éxito escolar que se promueve en todos los niños y a todas las edades, y los beneficios de metodologías de aprendizaje en las que se implica toda la comunidad de aprendizaje (MECD, 2015). Villalobos, Flórez y Londoño (2017) subrayan la importancia del acompañamiento familiar en el éxito del proceso de maduración de las capacidades intelectuales y sociales del estudiante, al tiempo que se consolida la calidad educativa.

La cooperación de ambos entornos en el desarrollo de la competencia emprendedora es fundamental $y$, aunque se prescribe en el actual marco legislativo, la observación de la práctica y los resultados de investigaciones recientes nos demuestran que su implementación es insuficiente y sigue siendo un reto pendiente.

El objetivo de este artículo es revisar el estado de la situación de la competencia emprendedora en la escuela; de la práctica internacional y nacional de la educación para el emprendimiento, y ofrecer una propuesta de práctica exitosa de desarrollo de la competencia emprendedora, basada en la sinergia familia-escuela, por la incidencia directa que estas acciones tienen en el futuro desempeño laboral de nuestros estudiantes y su vida en sociedad. 


\section{La competencia emprendedora en la escuela}

“Emprender” es un concepto amplio, polisémico (Damián, 2013), extenso y complejo, que carece de cualquier noción de linealidad (Neck y Greene, 2011). Actualmente no existe una definición de emprendimiento estándar (Paños, 2017). Unos autores hablan de actitud, rasgo de personalidad, intención; otros de orientación emprendedora, autoeficacia, actividad, y hay quien opta por los conceptos de capacidad, habilidad y competencia emprendedora. Sin embargo, un análisis de frecuencia de uso de este término nos permite afirmar que la competencia emprendedora, junto con los rasgos de personalidad de un emprendedor, son los aspectos más trabajados e investigados (Caballero-García et al., 2017b).

Generalmente, al término "competencia emprendedora" se le han unido dos interpretaciones (Sobrado y Fernández, 2010): el dominio de capacidades y actitudes empresariales y la creación de empresas. Nuestro interés se centra en la primera acepción.

Tratando de establecer una definición que sirva de marco común, Alcaraz (2011) señala que una persona emprendedora tiene la capacidad de generar y establecer metas, así como de trabajar ardua y perseverantemente en su logro; crear e iniciar un negocio, utilizar sus competencias para optimizar recursos o solucionar problemas de manera innovadora y aprovechar áreas de oportunidad que otros no han visualizado. A estos efectos, se entiende esta competencia como una habilidad específica para ofrecer respuestas no conocidas en un entorno en el que las necesidades económicas varían y aumentan (Núñez-Ladevéze, 2017).

El emprendimiento se considera motor de desarrollo y es un buen medio para generar riqueza y crear empleo en la creciente globalización (Minniti, 2012). Por tanto, las sociedades actuales necesitan emprendedores (Escat y Romo, 2015) y los gobiernos debieran invertir tiempo y recursos en educar a sus ciudadanos hacia el emprendimiento (González y Olivié, 2018).

La educación en emprendimiento tendrá repercusiones positivas en el dinamismo de las economías. Existe una relación positiva entre la aptitud hacia el emprendimiento y el progreso productivo (Núñez-Canal, 2017). El emprendimiento incluye un grupo de competencias que son fundamentales para la educación integral de los ciudadanos (Comisión Europea, 2016; Gómez-Núñez et al., 2017; Lackeus, 2013; Morris, Webb, Fu, y Singhal, 2013; Zubizarreta, Arellano y de León, 2014), como la capacidad de innovar, encontrar soluciones creativas y adaptarse al cambio (Jaramillo, 2008). Contribuir a la creación de nuevas empresas, ayudará a que los jóvenes sean más aptos para el empleo e intraemprendedores en su trabajo (Comisión Europea, 2014; González y Olivié, 2018). La competencia emprendedora en la escuela ha sido, años atrás, un reclamo indiscutible de nuestra sociedad para el cumplimiento de los retos de la globalización y las demandas socioeconómicas actuales. El liderazgo, la innovación y el emprendimiento son tan bien valorados por las empresas como los conocimientos técnicos y la formación específica (Béjar, 2017). La educación tiene una responsabilidad central en identificar emprendedores y fomentar el desarrollo de sus competencias emprendedoras (Krauss, 2011). 
La educación para el emprendimiento es esencial para el desarrollo de una cultura del emprendimiento en Europa (Eurydice, 2016). En los últimos años, la Unión Europea ha promovido en los estados miembros iniciativas para promover la cultura empresarial desde los sistemas educativos, considerando el fomento del espíritu emprendedor como algo fundamental en la creación de empleo, la mejora de la competitividad y el crecimiento económico (Alemany, Álvarez, Planellas y Urbano, 2011; Núñez-Ladevéze, 2017).

Desde principios del año 2000, la Organización para la Cooperación y el Desarrollo Económicos recomendó a los países miembros efectuar acciones tendientes a la incorporación de temas de emprendimiento en todos los niveles educativos (Damián, 2013).

En esa línea, el Consejo Europeo de Lisboa de marzo de 2000, definió la educación en el espíritu emprendedor como el motor para una cultura empresarial más dinámica. En febrero del año 2001, el Consejo de Ministros de Educación de la Unión Europea, promovió el desarrollo del espíritu de empresa en la educación y defendió el refuerzo de los vínculos entre instituciones educativas y empresas (Fernández y Reyes, 2017).

La Comisión Europea (2008, 2010, 2016) considera el emprendimiento como una competencia transversal, clave para todos los seres humanos y útil en todos los ámbitos de la vida (personal, social y profesional). En su Libro Verde define el emprendimiento como un modelo mental asociado a procesos de creatividad e innovación (Toca, 2010). En su «Plan de Acción sobre Emprendimiento 2020» (Comisión Europea, 2014), invitaba a los Estados miembros a que "garanticen que la competencia clave «emprendimiento» esté integrada en los planes de estudios de la enseñanza primaria y secundaria, la formación profesional, la enseñanza superior y la educación de adultos antes del final de 2015" (p.8). En su comunicación «Replantear la educación», insistió en la necesidad de incorporar el aprendizaje emprendedor en todos los sectores de la educación, incluido el no formal. Ambos documentos instan a los Estados miembros a proporcionar a todos los jóvenes una experiencia práctica de emprendimiento antes de finalizar la educación obligatoria.

En el contexto español, la Ley Orgánica 10/2002, de 23 de diciembre, de Calidad de la Educación (LOCE, 2002) incorporó por primera vez la competencia emprendedora en su articulado, indicando que "el espíritu emprendedor es necesario para hacer frente a la evolución de las demandas de empleo en el futuro" (p. 45189). Y cuatro años después, el concepto de emprendimiento, en la Ley Orgánica 2/2006, de 3 de mayo, de Educación (LOE, 2006). En ella quedó establecido como uno de los fines del sistema educativo español (art. 2.f): "El desarrollo de la capacidad de los alumnos para regular su propio aprendizaje, confiar en sus aptitudes y conocimientos, así como para desarrollar la creatividad, la iniciativa personal y el espíritu emprendedor" (p.15).

En educación infantil (EI) comenzó a evaluarse la capacidad para utilizar los recursos propios, el conocimiento de las posibilidades y limitaciones y la confianza para emprender nuevas acciones (Real Decreto 1630/2006, p. 478).

La Ley Orgánica 8/2013, de 9 de diciembre, para la Mejora de la Calidad Educativa (LOMCE, 2013) supuso un refuerzo para el desarrollo de la competencia emprendedora. En su Preámbulo I planteó la necesidad de que fuese apoyada por el sistema educativo y, en su Preámbulo VI, estableció entre sus objetivos "mejorar la empleabilidad y estimular el 
espíritu emprendedor de los estudiantes" (p.7). También modificó los objetivos de etapa que el alumnado debía alcanzar, tanto en Educación Primaria (EP) como en Educación Secundaria Obligatoria (ESO), promoviendo hábitos de trabajo en equipo, así como actitudes de confianza en uno mismo, sentido crítico, iniciativa personal, y desarrollo del espíritu emprendedor (Manso y Thoilliez, 2015).

En EP, la LOMCE especifica que, sin perjuicio de su tratamiento específico en algunas de las áreas de la etapa, el emprendimiento y la educación cívica y constitucional se trabajarán en todas ellas. En los cursos quinto y sexto, el área de "Creatividad y Emprendimiento" pretende que los alumnos sean capaces de conocer y explorar los límites de sus propias fortalezas y generen cambios en su entorno, agregando valor a su comunidad a través de proyectos de innovación y emprendimiento. El emprendimiento y la creatividad son considerados como "la capacidad para construir y transformar las circunstancias y el entorno en el que nos desenvolvemos, hacen referencia a un ejercicio constante de "crear valor" cualquiera que sea el contexto: personal, social o de negocios" (p.26). El alumno debe incorporarlas como una práctica propia habitual en su vida y en su ejercicio profesional.

Los currículos de ESO y Bachillerato (Real Decreto 1105/2014), también incorporaron elementos orientados a la autonomía personal; desarrollo y afianzamiento del espíritu emprendedor; y la adquisición de competencias para la creación y desarrollo de diversos modelos de empresas, que incluyen un 'Plan económico-financiero', fomento de la igualdad de oportunidades, respeto al emprendedor y al empresario, y ética empresarial.

La inclusión de la competencia emprendedora fue tan importante que, incluso el MECD insistió en que "sería recomendable plantear en un futuro un estudio que permitiera precisar con más detalle cuál es el estado del emprendimiento en los diferentes Grados y Máster de formación inicial del profesorado" (Diego y Vega, 2016, p.34). De esa manera, se dotaba al sistema educativo de profesionales capacitados para inculcar y motivar ese espíritu emprendedor.

Sin embargo, la cultura española no apoya la iniciativa emprendedora (Núñez-Canal, 2017). La tarea de orientar la educación al emprendimiento está aún en una etapa de "juventud" (Sánchez, 2013). Aunque ha mejorado, no es suficiente (Alemany et al., 2011).

El informe elaborado por la Red Global Entrepreneurship Monitor (GEM) España (Peña, Guerrero, González-Pernía y Montero, 2018) sostiene que la tasa de emprendimiento español se recupera y ha aumentado un punto con respecto al año anterior (del 5,2\% al 6,2\%) y, por primera vez en 8 años, ha superado el umbral del $6 \%$, acercándose a cifras anteriores a la crisis (7,6\% en 2007). A pesar de ello, el índice español sigue estando por debajo de la media europea (8,1\%). El emprendimiento es todavía "asignatura pendiente" y “siempre pospuesta tarea” en nuestras escuelas (Núñez-Ladevéze, 2017).

Entre los obstáculos a la iniciativa emprendedora se mencionan la falta de cultura emprendedora, el miedo al fracaso, la insuficiente formación en emprendimiento y el acceso a financiación. Núñez-Canal (2017) añade el desinterés del profesorado e incluso del propio sistema. 
Aun así, los expertos consideran necesaria una movilización social para construir una sociedad con más iniciativa emprendedora y un cambio en el sistema educativo español, que se adapte a las nuevas realidades, la favorezca y valore (Alemany et al., 2011; NúñezCanal, 2017). Béjar (2017) habla de la necesidad de un proyecto de emprendimiento, saber gestionar el talento y atreverse a dar el paso a la acción. Es aquí donde la familia adquiere su verdadero protagonismo y co-responsabilidad. La colaboración desde el núcleo familiar y la comunidad dará a los alumnos motivación extrínseca de sus más allegados, y puntos de vista relacionados de sus dos principales modelos de referencia, lo que se convertirá en motivación intrínseca para la acción.

\section{Prácticas de educación para el emprendimiento. Estado de la situación a nivel internacional y nacional.}

En nuestra revisión de la situación de la intervención sobre el emprendimiento en la escuela, hemos podido comprobar que la mayor parte de los planes contienen dos finalidades claras: promover la cultura emprendedora en los procesos formativos, facilitando y apoyando las iniciativas emprendedoras; y propiciar mejoras permanentes del sistema educativo en las que crear, innovar y emprender sean consecuencia inherente a los procesos educativos en todos los niveles.

Diez elementos clave conforman una estrategia modélica de educación para el emprendimiento en cualquier programa educativo (Comisión Europea, 2010): 1) Compartir una definición clara de educación emprendedora; 2) potenciar la cooperación interministerial en la articulación de la estrategia; 3) consultar y comunicarse con los principales interlocutores para lograr el mayor grado de comprensión y difusión del concepto; 4) incluir el espíritu emprendedor como competencia clave en todas las áreas del currículo; 5) plantear objetivos visionarios y ambiciosos; 6) integrar y apoyar la estrategia con ejemplos de buenas prácticas; 7) formar al profesorado; 8) desarrollar un modelo lógico que incluya indicadores, resultados e impacto esperado; 9) crear marcos de progresión desde la EP hasta la educación superior, y 10) destinar fondos para desarrollar la estrategia.

El informe Eurydice (2016) muestra que, en EP, aproximadamente la mitad de los países tienen un enfoque transversal, sin que exista una vinculación directa con materias concretas. En catorce sistemas educativos, la educación para el emprendimiento se encuentra integrada en materias obligatorias. En cuatro países (España, Eslovenia, Finlandia y Noruega), combinadas con objetivos transversales, y es poco frecuente encontrarla como optativa o integrada en materias optativas.

En ESO, la educación para el emprendimiento es más habitual que en EP y se imparte con una variedad de enfoques: transversal, obligatorio y optativo. Con frecuencia es, tanto una asignatura separada como una materia integrada dentro de otras, particularmente en el ámbito de las ciencias sociales, la economía y las ciencias empresariales, o parte de materias opcionales. En la Formación Profesional (FP), el emprendimiento representa una cifra inferior a la de primaria y secundaria general, y datos similares a la secundaria superior en los cursos especializados. 
La educación para el emprendimiento de todos los países cubre los objetivos de ciudadanía activa, emprendimiento social, creación de empresas y, de manera especial, la empleabilidad. En España, las asignaturas obligatorias trabajan la creatividad y el emprendimiento mejorando habilidades relacionadas con la información, desarrollo de ideas y presentación de conclusiones innovadoras (ciencias sociales), así como autonomía y capacidad de emprendimiento para conseguir logros personales y responsabilizarse del bien común (valores cívicos y sociales).

Los principales métodos de aprendizaje son: trabajo por proyectos, retos prácticos, retos de la comunidad y creación de miniempresas o iniciativas microfinanciadas. De todos ellos, los ejemplos más generalizados son la creación de miniempresas y el trabajo por proyectos. Muy pocos países contemplan las experiencias prácticas de emprendimiento como parte regular y obligatoria de su currículo oficial. En la mitad de los países/regiones son opcionales. Muchas de ellas se realizan con colaboradores externos, como Junior Achievement, que se ha convertido en una de las mayores organizaciones internacionales impulsoras de la formación emprendedora, la educación financiera y la preparación laboral a nivel mundial, aportando experiencias, métodos, materiales y herramientas de autoevaluación. Sus actividades están consideradas como "Best practice" por la Unión Europea.

Más de la mitad de los países ofrecen poca o ninguna orientación didáctica en materia de métodos de enseñanza, siendo más habituales en ESO y FP. El aprendizaje activo y las actividades fuera del aula son las más frecuentes, siendo la menos común el aprendizaje experimental. Tampoco se estima como acción prioritaria la determinación y evaluación de resultados de aprendizaje.

Existen centros de formación y redes de comunicación entre profesores. Sin embargo, la situación podría mejorar si existiera un mayor compromiso por parte de las autoridades centrales.

Más de la mitad de los países europeos destinan fondos al desarrollo e implementación de la educación para el emprendimiento. No obstante, está pendiente de desarrollo en toda Europa una financiación estable y a largo plazo que haga posible un enfoque integral.

A nivel nacional, solo tres Comunidades Autónomas (Andalucía, Cantabria y Galicia) cuentan con estrategias y planes específicos de educación para el emprendimiento. El resto ha optado por estrategias amplias de apoyo a la creación y consolidación de empresas (Diego y Vega, 2016).

La educación para el emprendimiento se integra en el currículo de tres maneras: como elemento transversal en todas las etapas (sentido de la iniciativa y emprendimiento), integrándola en materias existentes o creando materias específicas (se concentran en ESO, Bachillerato y FP). En todas estas experiencias se repite un patrón que hace hincapié en distintas facetas como son la personal, social, cultural o empresarial, creando un continuo de oportunidades en las sucesivas etapas del sistema educativo.

Sin embargo, el informe RediE 2015 (Diego y Vega, 2016) concluye que no disponemos de una estrategia de educación emprendedora generalizada, y contamos con una participación 
muy desigual de los diversos grupos de interés, por lo que no existen modelos claros de progresión para el desarrollo de la competencia, faltando, además, una visión de conjunto para los criterios de evaluación y estándares de aprendizaje iniciada con la LOMCE (2013). En consecuencia, adolecemos de una coherencia y complementariedad entre materias y una progresión clara entre niveles, siendo éstos dos aspectos manifiestamente mejorables. Se detectan también carencias en la formación del profesorado, estando el emprendimiento prácticamente ausente en su formación inicial. La creación de empresas, en contraposición con muchos otros países europeos, no está reconocida como un itinerario formativo específico y, en todo caso, es la FP quien ofrece más apoyo a los futuros emprendedores. Queda, por tanto, mucho por hacer.

\section{Propuesta de participación familiar para el desarrollo de la competencia emprendedora en la escuela y la empleabilidad del alumnado}

La transformación de la educación no depende sólo del sistema educativo, sino de toda la sociedad y, en especial, del núcleo familiar, quien debe, y así lo dispone la ley (LOMCE, 2013), asumir un papel activo.

La relación familia-escuela coge fuerza y se ve impulsada por el marco legislativo desde los primeros cursos de El. En el Real Decreto 1630/2006 de 29 de diciembre, por el que se establecen las enseñanzas mínimas del segundo ciclo de esta etapa, se habla de la familia y la escuela como "los primeros grupos sociales de pertenencia" (p.479) y de la especial relevancia que tiene para el proyecto educativo "la participación y colaboración con las familias" (p. 474). En el preámbulo II de la actual ley educativa (LOMCE, 2013), leemos "Las familias son las primeras responsables de la educación de sus hijos y por ello el sistema educativo tiene que contar con la familia y confiar en sus decisiones" (p.4).

La coordinación familia-escuela es fundamental para el desarrollo de la competencia emprendedora. Ambas desempeñan roles educativos complementarios y deben coordinarse y entenderse para que el niño, como sujeto de educación, pueda hacer uso de un desarrollo dinámico, una educación completa y de alta calidad humana y cultural (Cano y Casado, 2015). El involucramiento de la familia es uno de los predictores más potentes del éxito escolar (Boonk, Gijselaers, Ritzen y Brand-Gruwel, 2018; Grant y Ray, 2013; Jeynes, 2011; Rasbash, Leckie, Pillinger y Jenkins, 2010).

Autores como Davidson y Honig (2003) han demostrado que es mucho más probable que se atreva a ser emprendedor un individuo cuyos padres, amigos y vecinos también lo son o animan a ello. Si la familia trabaja conjuntamente con la escuela en el desarrollo de la competencia emprendedora, aseguraremos que los jóvenes tengan las herramientas necesarias para crecer tomando sus propias decisiones laborales y siendo conscientes de sus posibilidades en el mercado laboral. Forma parte del aprender a emprender.

Pero no sólo queda en manos de la familia y la escuela este propósito, García Sanz et al. (2016) proponen la comunidad como un eslabón más de la cadena educativa. Los programas educativos que involucran a las familias y la comunidad no solo estimulan el 
éxito académico de los estudiantes, también mejoran la inclusión social y la inserción laboral (Salimbeni, 2011).

En coherencia con lo argumentado hasta ahora, nuestra propuesta-modelo tendría como objetivo general generar cultura de emprendimiento en el centro y en cada etapa educativa y, como objetivos específicos, desarrollar la competencia emprendedora del alumnado, involucrando a la familia y con proyección hacia la comunidad, para que el alumno detecte fortalezas y debilidades en ese contexto real, que le ayuden a desarrollar su espíritu crítico y comunicativo, entre otros, y a ofrecer propuestas propias que potencien su autonomía y creatividad, como producto final.

La formación que proponemos podría estructurarse en torno a seis módulos de contenidos: Ciudadanía activa y emprendimiento social; empleabilidad; motivación de logro; Inteligencia emocional y Liderazgo; Creatividad y generación de ideas; Planificación, Desarrollo y Evaluación de un plan de emprendimiento, que se trabajarían con un enfoque transversal y, en su defecto, como materia concreta obligatoria para cada etapa educativa, siguiendo las directrices de Eurydice (2016).

Sería fundamental que la acción pedagógica se adaptase al contexto educativo y a la etapa educativa a la que va dirigida, que fuera rigurosa y de carácter práctico, al tiempo que se acompañara de procesos de seguimiento y mentoring, por parte del profesorado y la familia, que asegurara una acción de apoyo mutuo, eficaz y efectiva, progresiva, duradera y mejorable en el tiempo.

Sugerimos una metodología activa, alineada con la práctica internacional (Eurydice, 2016), que tome como referencia el sistema de trabajo por proyectos, retos prácticos y de la comunidad, y se desarrolle por medio de actividades prácticas, basadas en el aprendizaje participativo y cooperativo (Béjar, 2017; Comisión Europea, 2010), en las que los estudiantes internalizarían los diferentes contenidos del programa de manera individual o en grupo, y en el entorno de una verdadera comunidad de aprendizaje (MECD, 2015), con estrategias de búsqueda de información, diseño de proyectos, y juegos de cambios de rol, que les permitan enfrentarse a una situación real, dentro del contexto habitual donde se desenvuelven, y les induzca a unas ideas y pensamientos que difieran de los suyos propios, y donde puedan abrirse vías hacia el ámbito laboral. La secuencia de actividades que proponemos puede seguir la lógica "de lo general a lo específico" y la dinámica de la investigación-acción, con procesos de observación, reflexión, diseño, puesta en práctica y revisión-mejora.

Autores como Jaén et al. (2013), Ramírez y Fuentes (2013) han demostrado que actividades que ayudan a los alumnos a enfrentarse a situaciones de la vida real, combinadas con metodologías de innovación que fomentan la creatividad y favorecen la implicación y motivación de los estudiantes, ayudan al desarrollo personal y profesional, así como mejoran el rendimiento académico de los alumnos y desarrollan su espíritu emprendedor. "Aprender a tener mediante actividades de emprendimiento" de Javier Damián Simón (2012); el "Programa Junioremprende" de Augusto Andrade Díaz (2013), "Mi Primera Empresa: emprender jugando" de la FESE (2011); el "Método del YPD BOX" de Felipe Hernández Perlines (2017), son algunos ejemplos-referencia de prácticas de éxito en 
educación emprendedora para las etapas educativas de primaria y secundaria, basadas en la simulación, el juego, la creación de miniempresas y uso de las TIC como recurso para trabajar la competencia emprendedora, el cambio educativo y la transformación social.

Como producto final, los alumnos realizarían un plan de negocio (adaptado a sus circunstancias y las de su contexto), para el que será necesaria la puesta en práctica de habilidades de análisis del entorno (que detecten necesidades, amenazas y oportunidades de mejora), búsqueda de información, análisis e interpretación de datos y la realización de un proyecto-empresa realista (con la ayuda de los compañeros y las orientaciones de su profesor) que incluya unos objetivos concretos, una metodología cooperativa y de investigación, una previsión de recursos (espaciales, humanos y económicos), unos mecanismos de seguimiento, evaluación y mejora, una temporalización y un plan de promoción y marketing, que será defendido en clase y evaluado empleando diferentes estrategias (autoevaluación, coevaluación y heteroevaluación) y criterios de evaluación (centrados en la capacidad de búsqueda y análisis de datos, diseño de instrumentos, habilidad comunicativa, nivel de autonomía y creatividad, utilidad y viabilidad de la propuesta). La familia estaría implicada en todas las fases del proceso. De este modo, aseguraríamos el acompañamiento familiar (Villalobos et al., 2017), y que los alumnos recibieran de sus entornos más cercanos sus primeras experiencias de emprendimiento (Romero y Espasandín, 2016).

\section{Conclusiones}

El emprendimiento es el primer acercamiento de los estudiantes al mundo empresarial y les prepara para la vida en sociedad. Contribuye, por tanto, a su desarrollo personal, social y laboral (Caballero-García et al., 2017a, 2017b; Comisión Europea, 2016; Ramírez y Fuentes, 2013). De ahí la importancia de su progresiva inclusión en la escuela, y más específicamente en aquellos momentos del sistema educativo en los que los alumnos toman opciones de estudios en función de su interés profesional (Ramírez y Fuentes, 2013).

Aunque la mayoría de los países afirman haber integrado la educación para el emprendimiento en su currículo, ninguno la ha integrado plenamente y de manera sistemática en sus centros (Béjar, 2017), en todos los alumnos, ni en todos los niveles, garantizando una enseñanza de alta calidad. Las investigaciones muestran niveles generalmente bajos de participación en el aprendizaje para el emprendimiento en los centros educativos y la necesidad de seguir desarrollando estas destrezas en los jóvenes (Eurydice, 2016).

En nuestra revisión bibliográfica hemos evidenciado las carencias y debilidades de las que adolece nuestro actual sistema educativo en materia de emprendimiento, lo que nos suscita la necesidad de líneas de trabajo que ayuden a completar las estrategias y programas que actualmente se implementan y su evaluación.

El objetivo de este artículo ha sido revisar la bibliografía más significativa sobre el estado de la competencia emprendedora en la escuela, y de las prácticas educativas internacionales y nacionales, con el fin de entenderlo en su perspectiva más global y ofrecer una propuestamodelo de cómo desarrollar la competencia emprendedora desde una perspectiva común, 
impulsada por la escuela y la familia, y con el apoyo institucional y social que necesita para asegurar el éxito y la trascendencia de sus resultados.

Nuestra propuesta recoge las recomendaciones europeas de promover la cultura y las iniciativas emprendedoras y su mejora, desde un enfoque integral y transversal, como parte estratégica regular y obligatoria del currículo oficial, y con itinerarios formativos específicos por etapas educativas. En materia de métodos de enseñanza, promueve el aprendizaje activo y experiencial, orientado a la comunidad. Prepara para la vida adulta descubriendo y experimentando un camino único y vivencial, cuyo desemboque es la inclusión personal en el mercado laboral. Y, en términos de mejora, contempla criterios formales de evaluación y estándares de aprendizaje.

El hecho de formar jóvenes más activos, capaces de crear proyectos y llevarlos a cabo, no solo les beneficia en su propio éxito (MECD, 2015; Villalobos et al., 2017), sino que también les ayuda a crear una sociedad más emprendedora e innovadora (De la Torre et al., 2015; Eurydice, 2016).

Todo ello se consigue a través de la construcción de una cultura emprendedora que sirva de puente entre núcleo familiar y centro escolar que lo apoye, puesto que es un proceso de reinvención continuo, basado en la innovación (González y Olivié, 2018).

La cultura de emprendimiento es un modo mental de pensar y actuar que predispone a las personas a descubrir necesidades y oportunidades en su entorno social. El emprendimiento impulsa a las personas a salir de su zona de confort y a poner en marcha un plan de acción de mejora del bienestar personal y social (Béjar, 2017).

Proponemos que los programas que desarrollan y estimulan la creatividad y el espíritu emprendedor empiecen a edades tempranas y continúen hasta edades adultas en los entornos universitarios, donde el alumnado se ve envuelto en una formación continua para su inminente inmersión en el mercado laboral. De este modo, las actuaciones concretas en el sistema educativo se verán vinculadas con la participación de las familias y la comunidad educativa, integrando el emprendimiento en la vida de los jóvenes, tanto en el currículo como en su vida cotidiana, con el objetivo de garantizar que los jóvenes adquieran esta competencia de manera coherente y progresiva hasta su inclusión en el mercado laboral.

Los centros educativos siempre han sido mediadores en la transición a la etapa adulta y profesional. Hoy también se espera que puedan desempeñar con intensidad esta función social. Pero han de adecuarse a las actuales circunstancias. Se necesita acercar las escuelas a las empresas. Pero esta aproximación no es posible sin una apertura al cambio. Necesitamos urgentemente nuevos modelos formativos, más abiertos, participativos, y emprendedores (Béjar, 2017).

Nos enfrentamos a un reto educativo, el de perseverar en la capacidad de mantener el espíritu emprendedor a lo largo de las diferentes etapas escolares, evolucionar y reinventar el rol educativo familiar-escuela-comunidad. 


\section{Referencias}

Alcaraz Rodríguez, R.E. (2011). Perfil del Emprendedor, un estudio comparativo entre estudiantes de países de Iberoamérica. Propuesta de un modelo formativo universitario de emprendedores a partir del diseño de un instrumento de medición de características emprendedoras. Tesis doctoral. San Sebastián: Universidad de Deusto.

Alemany, L., Álvarez, C., Planellas, M. y Urbano, D. (2011). Libro Blanco de la iniciativa emprendedora en España. Barcelona: Fundación Príncipe de Gerona-ESADE.

Andrade Díaz, A. (2016). La importancia de la iniciativa emprendedora en la educación primaria como método para el fomento de las competencias básicas. Tesis Doctoral. Badajoz: Universidad de Extremadura.

Arnaiz, A. (2015). La cultura emprendedora en la educación secundaria. Tesis doctoral. Oviedo: Universidad de Oviedo.

Béjar, M. (2017). Neuroemprendimiento. Revista Padres y Maestros, (369), 64-70.

Boonk, L., Gijselaers, H.J.M., Ritzen, H. y Brand-Gruwel, S. (2018). A review of the relationship between parental involvement indicators and academic achievement. Educational Research Review, 24, 10-30.

Caballero-García, P.A., Guillén-Tortajada, E. y Jiménez-Martínez, P. (2017a). El emprendimiento como propuesta de cambio y herramienta clave para acercar la formación universitaria a la realidad social del mercado laboral. En Simposio Internacional "El desafío de emprender en la escuela del siglo XXI" (SIEMPRE 2017) (pp. 2-21), diciembre 18-19. Sevilla, España: Universidad de Sevilla.

Caballero-García, P.A., Guillén-Tortajada, E. y Jiménez-Martínez, P. (2017b). Creativity, entrepreneurship and happiness: ingredients for a Higher Education change. 21st World Multi-Conference on Systemics, Cybernetics and Informatics (WMSCl 2017). Proceedings, (1), 318-322.

Cano, R. y Casado, M. (2015). Escuela y familia. Dos pilares fundamentales para unas buenas prácticas de orientación educativa a través de las escuelas de padres. Revista Electrónica Interuniversitaria de Formación del Profesorado, 18(2), 15-27.

Comisión Europea (2008). Entrepreneurship in higher education, especially in non-business studies. Bruselas: Comisión Europea.

Comisión Europea (2010). Towards Greater Cooperation and Coherence in Entrepreneurship Education. Bruselas: Comisión Europea.

Comisión Europea (2014). Educación en emprendimiento. Guía del educador. Bruselas: Comisión Europea.

Comisión Europea (2016). EntreComp: The Entrepreneurship Competence Framework. Bruselas: Comisión Europea.

Damián Simón, J. (2013). Sistematizando experiencias sobre educación en emprendimiento en escuelas de nivel primaria. Revista Mexicana de investigación educativa, 18(56), 159-190.

Davidson, P. y Honig, B. (2003). The role of social and human capital among nascent entrepreneurs. Journal of Business Venturing, 18(3), 301-331. 
De la Torre Cruz, T., Ruiz Palomo, E., Escolar Llamazares, M., Baños Martínez, V., Gañán Ibáñez, Á., Corbí Santamaría, M., Santos González, J., y Sánchez, A. (2015). Impacto de la educación en la cultura del emprendimiento: Estudio cualitativo. International Journal of Developmental and Educational Psychology, 2(1), 117-128.

Diego, I. y Vega, J. A. (2016). La educación para el emprendimiento en el sistema educativo español: año 2015. Estudio redIE. Madrid: MECD, Secretaría General Técnica. Escat, M. y Romo, M. (2015) Emprendimiento y personalidad creativa en estudiantes universitarios. Creatividad y Sociedad, 23, 64-99.

Eurydice (2016). La educación para el emprendimiento en los centros educativos en Europa. Luxemburgo: Oficina de Publicaciones de la Unión Europea. doi:10.2797/301610

Fernández Batanero, J.M. y Reyes Rebollo, M.M. (2017). Competencias emprendedoras del alumnado de educación permanente de Andalucía. Percepción del profesorado. Educación XXI, 20(1), 253-275.

FESE (2011). Manual educativo para asesores. Mi Primera Empresa: "emprender jugando". México: Fundación Educación Superior Empresa-ANUIES.

García-Sanz, M.P., Hernández-Prados, M.A., Parra-Martínez, J. y Gomariz-Vicente, M.A. (2016). Participación familiar en la etapa de educación primaria. Perfiles Educativos, 38(154), 97-117.

García-Zárate, M.L. (2013). La caracterización de la cultura escolar de una escuela normal a través de su normativa, ceremonias y valores. Tesis doctoral. México, Instituto Tecnólogico y de Estudios Superiores de Occidente.

Gómez-Núñez, L., Llanos Martínez, M., Hernández Rico, T., Mejía Rodríguez, D., Heilbron López, J., Martín Gallego, J., Mendoza Soto, J. y Senior Roca, D. (2017). Competencias emprendedoras en Básica Primaria: Hacia una educación para el emprendimiento. Pensamiento \& Gestión, (43), 150-188

González, E. y Olivié, C. (2018). Empresa familiar, emprendimiento e intraemprendimiento. Madrid: EAE Business school.

Grant, B.K. y Ray, J.A. (2013). Home, Shool, and Community Collaboration. London: Sage.

Hernández-Perlines, F. (2017). Impacto del aprendizaje del emprendimiento a través de las TIC. IJERI: International Journal of Educational Research and Innovation, (8), 128-147.

Jaén, I., Moriano, J.A. y Liñán, F. (2013). Personal values and entrepreneurial intention: an empirical study. En A. Fayolle, P. Kyrö, T. Mets y U. Venasaar (Eds.), Conceptual Richness and Methodological Diversity in Entrepreneurship Research. Cheltenham: Edward Elgar.

Jaramillo, L. (2008). Emprendimiento: Concepto básico en competencias. Revista Lumen, 7. Recuperado de http://guayacan.uninorte.edu.co/divisiones/iese/lumen/ediciones/7/ articulos/emprendimiento.pdf

Jeynes, W.H. (2011). Parental Involvement and Academic Success. New York: Routledge.

Krauss, C. (2011). Actitudes emprendedoras de los estudiantes universitarios: El caso de la Universidad Católica del Uruguay. Dimensión empresa, 9(1), 28-40.

Lackeus, M. (2013). Developing Entrepreneurial Competencies: An Action-Based Approach and Classification in Education. Tesis doctoral. Suiza: Chalmers University of technology. 
León Tamayo, A.L. y Barrera Sánchez, L.D. (2017). El bienestar psicológico y el rendimiento académico en los estudiantes de la escuela de psicología de la Pontificia Universidad Católica del Ecuador Sede Ambato. Proyecto de Investigación. PUCESA Repositorio.

Ley Orgánica 10/2002, de 23 de diciembre (LOCE, 2002), de Calidad de la Educación. Boletín Oficial del Estado, (307), de 24 de diciembre, 45188-45220.

Ley Orgánica 2/2006, de 3 de mayo (LOE, 2006), de Educación. Boletín Oficial del Estado, (106), de 4 de mayo, 1-110.

Ley Orgánica 8/2013, de 9 de diciembre (LOMCE, 2013), para la mejora de la calidad educativa. Boletín Oficial del Estado, (295), de 10 de diciembre, 97858- 97921.

Manso, J. y Thoillez, B. (2015). La competencia emprendedora como tendencia educativa supranacional en la Unión Europea. Bordón. Revista de Pedagogía, 67(1), 85-100.

Martínez Méndez, S. (2007). El uso de las TIC como recurso didáctico para el fomento del espíritu emprendedor. En S. Marín Hernández, F. Ballina Ríos y J. Tagua Gómez (Coords.). Las competencias profesionales relacionadas con las TIC y el espíritu emprendedor. Madrid: MECD, Subdirección General de Información y Publicaciones.

MECD. Ministerio de Educación Cultura y Deporte (2015). La participación de las familias en la educación escolar. Madrid: Secretaria General Técnica.

Minniti, M. (2012). El emprendimiento y el crecimiento económico de las naciones. Economía industrial, 383, 23-30.

Morris, M., Webb, J., Fu, J., y Singhal, S. (2013). A Competency-Based Perspective on Entrepreneurship Education: Conceptual and Empirical Insights. Journal of Small Business Management, 51(3), 352-369.

Navarro Domenichelli, R. y Torregrosa Sahuquillo, D. (2012). Animación y motivación para el autoempleo: el espíritu emprendedor en la escuela. Revista Anuari de Psicologia de la Societat Valenciana de Psicologia, 13(1), 95-112.

Neck, H.M. y Greene, P.G. (2011). Entrepreneurship Education: Known Worlds and New Frontiers. Journal of Small Business Management, 49(1), 55-70.

Núñez-Canal, M. (2017). La educación emprendedora en la enseñanza escolar en España. Madrid: Universitas.

Núñez-Ladevéze, L. (2017). La importancia de fomentar una educación emprendedora en España desde las aulas. Diario de Análisis, Reflexión y valores. Recuperado de https://eldebatedehoy.es/cultura/libros/educacion-emprendedora

Núñez-Ladevéze, L. y Núñez-Canal, M. (2016). Noción de emprendimiento para una formación escolar en competencia emprendedora. Revista Latina de Comunicación Social, 71(9), 1069-1089.

Orrego, C. (2009). La fenomenología y el emprendimiento. Pensamiento y gestión, 27, 235252.

Paños Castro, J. (2017). Educación emprendedora y metodologías activas para su fomento. Revista Electrónica Interuniversitaria de Formación del Profesorado, 20(3), 33-48. doi: http://dx.doi.org/10.6018/reifop.20.3.272221

Peña, I., Guerrero, M., González-Pernía, J.L. y Montero, J. (2018). GEM, Global Entrepreneurship Monitor. Informe España. 2017-2018. Santander: Universidad de Cantabria. 
Ramírez, P. y Fuentes, C. (2013). Felicidad y Rendimiento Académico: Efecto Moderador de la Felicidad sobre Indicadores de Selección y Rendimiento Académico de Alumnos de Ingeniería Comercial. Formación Universitaria, 6(3), 21-30.

Rasbash, J.R., Leckie, G.B., Jenkins, J. y Pillinger, R.J. (2010). Children's educational progress: Partitioning family, school and area effects. Journal of the Royal Statistical Society, 173(3), 657 - 682.

Real Decreto 1630/2006, de 29 de diciembre, por el que se establecen las enseñanzas mínimas del segundo ciclo de Educación Infantil. Boletín Oficial del Estado, 4, de 4 de enero de 2007, 474-482.

Real Decreto 1105/2014, de 26 de diciembre, por el que se establece el currículo básico de la Educación Secundaria Obligatoria y del Bachillerato. Boletín Oficial del Estado, 3, de 3 de enero de 2015, 1-35.

Romero, R. y Espasandín, F. (2016). Iniciativa personal y emprendedora del alumnado de Primaria y 1er Ciclo de Secundaria: Aspectos personales, familiares y escolares. Intangible Capital, 12(5), 1221-1254.

Salimbeni, O. (2011). Escuela y comunidad: Participación comunitaria en el sistema escolar. Tendencias pedagógicas, (17), 19-31.

Sánchez, J.C. (2013). The Impact of an Entrepreneurship Education Program on Entrepreneurial Competencies and Intention. Journal of Small Business Management, 51(3), 447-465.

Santamaría Escobar, A.E., Moreno Arias, E.M., Torres Torres, E.F. y Cadrazco Parra, W. (2013). La influencia de le familia en el emprendimiento empresarial - el caso de tres pymes de los sectores comercial y de servicios de le ciudad de Sincelejo Sucre. Pensamiento Gerencial, 1. Recuperado de https://revistas.unisucre.edu.co/ index.php/rpg/article/view/86/97

Sobrado Fernández, L., y Fernández Rey, E. (2010). Competencias emprendedoras y desarrollo del espíritu empresarial en los centros educativos. Educación XXI, 13(1), 1538.

Toca Torres, C. (2010). Consideraciones para la formación en emprendimiento: explorando nuevos ámbitos y posibilidades. Revista Estudios Gerenciales, 26,41-60.

Uribe Toril, J., Valenciano, J.P. y Bonilla Martínez, J.J. (2013). El desarrollo local y empresarial: La formación como factor básico para orientar al emprendedor. Intangible Capital, 9(3), 644-677.

Villalobos J.L., Flórez, G.A. y Londoño, D.A. (2017). La escuela y la familia en relación con el alcance del logro académico. La experiencia de la Institución Educativa Antonio José de Sucre de Itagüí (Antioquia) 2015. Revista Aletheia, 9(1), 58-75.

Zubizarreta, A.C., Arellano, P.R., y de León Sánchez, B. (2014). Education for Entrepreneurship: The case of Cantabria. Procedia Social and Behavioral Sciences, 139, 512-518. 